\title{
Diagnóstico de proliferación bacteriana intestinal en niños: evidencia experimental para sustentar el empleo de lactulosa en la prueba de hidrógeno y su validación como prueba tamiz
}

\author{
Evelyn Mendoza ${ }^{1}$, Carlos Crismatt ${ }^{2}$, Ramón Matos ${ }^{1}$, Olga Sabagh ${ }^{1}$, Mercedes Campo ${ }^{1}$, \\ Johana Cepeda ${ }^{1}$, Daniel Villanueva ${ }^{1}$ \\ 1 Grupo de Investigación en Bioquímica Patológica, GRUBIOPAT, Universidad Libre, Barranquilla, Colombia \\ 2 Centro de Gastroenterología y Endoscopia Pediátrica, Barranquilla, Colombia
}

Introducción. Un recuento superior a $10^{5}$ unidades formadoras de colonias por $\mathrm{ml}$ de aspirado duodenal se considera la "prueba de referencia" para el diagnóstico de proliferación bacteriana intestinal, a pesar de su caracter invasivo. La prueba de hidrógeno en el aliento, con lactulosa, se aplica como alternativa diagnóstica no invasiva, pero el fundamento para el empleo de lactulosa carece de solidez.

Objetivos. Aportar evidencia experimental para sustentar el uso de lactulosa en la prueba de hidrógeno y validarla frente al cultivo de aspirado duodenal.

Materiales y métodos. Para fundamentar el uso de lactulosa se comparó la cinética de la lactasa intestinal frente a lactulosa con su cinética frente a la lactosa. Con el fin de validar la prueba de hidrógeno, ésta se aplicó a 47 niños afectados por trastornos gastrointestinales y los resultados se compararon con los de la prueba de referencia. Mediante una tabla de contingencia se estimó la validez y con el índice kappa, la concordancia.

Resultados. La cinética mostró que la lactasa es 240 veces menos eficiente que la lactulosa. La sensibilidad de la prueba de hidrógeno fue de $85,7 \%(82,0 \%-89,4 \%)$, la especificidad de $90,9 \%$ (89,3\%-92,5\%), el valor diagnóstico positivo de $80 \%(76,5 \%-83,5 \%)$ y el valor diagnóstico negativo de 93,8\% (92,1\%-95,4\%). El índice kappa de 0,785 (0,54-0,95) indicó una importante concordancia entre las pruebas diagnósticas.

Conclusiones. Se aporta evidencia experimental para sustentar el uso de la lactulosa en la prueba de hidrógeno y se valida ésta frente al patrón de referencia como prueba tamiz para diagnosticar la proliferación bacteriana intestinal.

Palabras clave: infecciones bacterianas/diagnóstico, intestinos, lactulosa, lactasa, sensibilidad y especificidad.

Diagnosis of small intestinal bacterial overgrowth in children: the use of lactulose in the breath hydrogen test as a screening test

Introduction. The standard method for diagnosis of small intestinal bacterial overgrowth is a duodenal aspirate that produces a coliform count greater than $10^{5}$ colonic forming units $/ \mathrm{ml}$. Because this is an invasive procedure, the lactulose breath hydrogen test is considered as a non-invasive alternative. Better experimental support is required, however, for the routine use of lactulose in diagnosis.

Objective. Experimental evidence is provided to support the use of lactulose in the breath hydrogen test. Validation of the test is accomplished by comparison to the duodenal aspirate culture.

Materials and methods. A rational basis for the use of lactulose was established by a comparison of the kinetics of lactulose and lactose on intestinal lactase. For validation, the hydrogen test was applied to 47 children suffering from gastrointestinal disorders, and the results compared with aspirate culture counts. The validity of the hydrogen test was assessed by a contingency table and the degree of agreement established by the kappa index. 
Results. The kinetics data showed that lactase is 240 times less efficient in presence of lactulose than it is in presence of lactose. The sensitivity of the breath hydrogen test was $85.7 \%(82.0$ $89.4 \%)$, its specificity $90.9 \%(89.3-92.5 \%)$, the predictive positive value $80.0 \%(76.5-83.5 \%)$ and the predictive negative value $93.8 \%$ (92.1-95.4\%); the kappa index=0.785 (0.54-0.95) showed excellent agreement between the two diagnostic tests.

Conclusion. The results provided experimental support for the use of lactulose in the breath hydrogen test, and it was validated as screening test in the diagnosis of small intestinal bacterial overgrowth.

Key word. Bacterial infections/diagnosis, intestines, lactulose, lactase, small intestinal bacterial overgrowth, sensitivity and specificity.

En humanos, el intestino es estéril en el momento del nacimiento pero, luego, los alimentos y el contacto con el medio facilitan la instalación de bacterias en ese nicho ecológico. Mientras en el intestino delgado el número de bacterias es escaso $\left(10^{3}\right.$ a $10^{5}$ unidades formadoras de colonia por mililitro) (UFC/ml) y está representado por especies aerobias Gram positivas, en el intestino grueso la cantidad es superior $\left(10^{11}\right.$ a $10^{12}$ UFC/ $\mathrm{ml}$ ) y está representada por bacterias anaerobias Gram negativas (1-3). Sin embargo, en circunstancias especiales, tales como gastritis, diabetes, insuficiencia renal, aclorhidria y estasis intestinal, la población bacteriana en el intestino proximal aumenta; cuando el número de UFC es superior a $10^{5} / \mathrm{ml}$ de aspirado duodenal, se considera que hay proliferación bacteriana intestinal, también conocido como síndrome de intestino contaminado $(1,4,5)$. Por lo tanto, el recuento del número de UFC en el cultivo de aspirado duodenal es la forma de diagnosticar la proliferación bacteriana intestinal $y$, aunque invasiva, constituye la prueba de referencia $(1,6-8)$.

Cuando hay proliferación, desde luego, se aumenta la actividad metabólica bacteriana a expensas de los nutrientes procedentes de la dieta; así, aparecen productos que lesionan la mucosa intestinal, hay pérdida de electrolitos y se acumulan en el intestino gases como el gas carbónico $\left(\mathrm{CO}_{2}\right)$, el amoníaco $\left(\mathrm{NH}_{3}\right)$, el metano $\left(\mathrm{CH}_{4}\right)$ y el hidrógeno $\left(\mathrm{H}_{2}\right)(1,7)$. Éste, una vez

\footnotetext{
Correspondencia:

Daniel Villanueva, Facultad de Ciencias de la Salud, Universidad Libre, Km. 7 carretera a Puerto Colombia, Barranquilla.

Teléfono: 359 8866, extensión 149, fax: 3599782

danielvillanueva@unilibrebaq.edu.co,danvito@hotmail.com

Recibido: 23/11/06; aceptado: 19/04/07
}

absorbido, es llevado por la sangre hasta el pulmón, órgano que lo elimina en el aire de la espiración, a partir del cual puede ser cuantificado. He aquí el fundamento de la prueba de hidrógeno en el aliento, la cual se usa como criterio diagnóstico indirecto de proliferación bacteriana intestinal; en efecto, si después de la administración oral de un azúcar de prueba (lactulosa, glucosa, xilosa), se aumenta el hidrógeno en el aliento, por encima de 20 partes por millón (ppm) con respecto al valor basal, se infiere que las bacterias desdoblaron el azúcar y originaron dicho gas $(1,6,8)$.

La prueba de hidrógeno en el aliento -con lactulosa-, procedimiento no invasivo, se ha venido usando como alternativa diagnóstica de proliferación bacteriana intestinal $(9,10)$; sin embargo, la evidencia experimental que fundamenta el uso de la lactulosa no es consistente. Los objetivos de este estudio son: aportar evidencia experimental para darle solidez al fundamento del uso de lactulosa y validar la prueba de hidrógeno en el aliento, tras la administración oral de dicho azúcar, frente al cultivo de aspirado duodenal, considerado la "prueba de referencia".

\section{Materiales y métodos}

Reactivos y equipos. La glucosa oxidasa (E.C. 1.1.3.4), la peroxidasa (E.C. 1.11.1.7), la bgalactosidasa (E.C. 3.2.1.23), la galactosa deshidrogenasa (E.C. 1.1.1.48), el NAD, el 2-2 azino-di (3-etil benzotiazolino sulfónico) y el DLditiotreitol fueron comprados a Sigma (St. Louis Mo, USA); Ia DEAE celulosa (DE 52) fue comprada a Whatman (Springfield Mills, Inglaterra); el ultrogel ACA-34 se adquirió de Reactifs IBF Pharmindustrie (Francia); la lactosa, la galactosa, 
el reactivo de Folin Ciocalteau y el tritón X-100 se obtuvieron de Merck (Darmstad, Alemania); la lactulosa fue una donación de Laboratorios Chalver de Colombia.

Los equipos utilizados fueron: espectrofotómetro, Beckman DU ${ }^{\circledR}$ 640, Palo Alto California, USA; cromatógrafo de gases Microlyzer 12i de Quintron Instruments Co. Milwaukee, USA, y endoscopio Olympus XPE pediátrico.

Diseño metodológico. Este estudio consta de una parte experimental y de otra epidemiológica. La parte experimental in vitro apunta a darle soporte al uso de lactulosa y consiste en la comparación de la cinética de la lactasa intestinal frente a la lactulosa y la lactosa. La parte epidemiológica consiste en la validación de la prueba de hidrógeno en el aliento tras la administración oral de lactulosa, frente a la prueba de referencia: el cultivo de aspirado duodenal. Para ello, con base en los resultados de la prueba de referencia, se conformaron dos grupos de sujetos de observación, los enfermos y los sanos, a los cuales se les hizo la prueba de hidrógeno en el aliento. Con los datos resultantes se elaboró una tabla de $2 \times 2$ a partir de la cual se calcularon la sensibilidad, la especificidad y los valores diagnósticos positivo y negativo. Además, se determinó la concordancia entre las dos pruebas diagnósticas.

Obtención de la lactasa intestinal. La lactasa se obtuvo de la mucosa intestinal de un trozo de yeyuno de cordero, Ovis aries, la cual se raspó con una espátula y, luego, se homogeneizó con un politrón; a partir del homogeneizado, la enzima fue parcialmente purificada según el método descrito por Skovbjerg et al. (11). La actividad enzimática en las fracciones cromatográficas se determinó midiendo la glucosa, con glucosa oxidasa. El factor final de purificación fue de 50 .

Cinética comparada de lactasa frente a lactosa y frente a lactulosa. Inicialmente, se evaluó el porcentaje de hidrólisis de lactosa y de lactulosa mediante la determinación de la galactosa liberada; se hizo en alícuotas de $10 \mu \mathrm{l}$ tomadas a diferentes tiempos de un incubado $\left(37^{\circ} \mathrm{C}\right)$ que en un volumen de $200 \mu$ l contenía el sustrato, la solución tampón y la enzima. En la mezcla de reacción el sustrato y el maleato $\mathrm{pH} 6,0$ estaban a $80 \mathrm{mM}$ y las cantidades de enzima usadas fueron $10 \mu$ frente a lactosa y $120 \mu$ frente a lactulosa. Luego, se determinaron los parámetros cinéticos de la enzima mediante la representación de dobles recíprocos producto de los datos obtenidos cuando se incubó a $37^{\circ} \mathrm{C}$ la enzima frente a diferentes concentraciones de sustrato; los ensayos se hicieron con maleato $\mathrm{pH} 6,0,80 \mathrm{mM}$, $10 \mu \mathrm{l}$ de enzima y volumen final de $50 \mu \mathrm{l}$.

Determinación de la actividad de lactasa. La valoración de la actividad de la enzima se hizo mediante el método de Duodoroff (12), determinando la cantidad de galactosa liberada en la reacción. La unidad enzimática se definió como la cantidad de enzima que puede catalizar la conversión de $1 \mu \mathrm{mol}$ de sustrato en producto, en un minuto, en las condiciones especificadas.

Determinación de proteína. La determinación de proteína en los homogeneizados de mucosa y en las fracciones cromatográficas se hizo por el método descrito por Lowry (13).

Pacientes. Se trata de una muestra no probabilística y escogida a criterio, constituida por 47 niños con edades comprendidas entre 1 y 13 años (media $6 \pm 3,3$ años; todos tenían cuatro años o más, excepto uno), los cuales consultaron por trastornos gastrointestinales al Centro de Gastroenterología y Endoscopia Pediátrica de Barranquilla, durante el período de enero a junio de 2005.

Los criterios de inclusión fueron: a) ausencia, durante las dos últimas semanas, de tratamiento con antibióticos o cualquier otro agente terapéutico que pudiera haber alterado la motilidad o la microflora intestinal; b) dieta ligera y ausencia de ejercicio el día anterior a la prueba; c) ayuno de 10 horas, excepto el niño menor de cuatro años, quien ayunó durante 6 horas; d) consentimiento informado firmado por sus padres; e) presencia de bacterias, juzgada por tinción de Gram, en muestra de aspirado duodenal obtenida durante una endoscopia previamente indicada.

Durante este procedimiento, a los pacientes se les tomó una muestra de aspirado duodenal con el fin de hacer cultivo y recuento de bacterias. Según el resultado de la endoscopia, $11(23,3 \%)$ 
de los 47 pacientes no presentaban patología visible y $14(30 \%)$ presentaron duodenitis, 11 $(23,3 \%)$ gastritis, 5 (10,5\%) esofagitis, 5 (10,5\%) gastroduodenitis y uno $(2,1 \%)$ gastroduodenitis alérgica. De acuerdo con el recuento de UFC/ml de aspirado duodenal, los pacientes se clasificaron en dos grupos: niños con proliferación bacteriana intestinal ( $>10^{5} \mathrm{UFC} / \mathrm{ml}$ de aspirado duodenal) y niños sin proliferación bacteriana intestinal $\left(<10^{5}\right.$ UFC/ml de aspirado duodenal). Dos días después de la endoscopia, a cada paciente se le hizo la correspondiente prueba de hidrógeno en el aliento.

Obtención del aspirado duodenal. El aspirado duodenal se obtuvo con un endoscopio Olympus XPE pediátrico. Cuando se llegó a la segunda porción del duodeno, se introdujo la sonda estéril a través del canal de biopsia del endoscopio y se aspiró el líquido por medio de una jeringa, también estéril, de $60 \mathrm{ml}$; una parte del líquido se pasó a un tubo con caldo tioglicolato prerreducido para el estudio de anaerobios, y la restante se usó para la cuantificación y el estudio de aerobios y de facultativos.

Recuento de bacterias en el aspirado duodenal. Las muestras de aspirado duodenal se sembraron en agar sangre y en forma masiva, $10 \mu \mathrm{L}$ de la muestra original y $10 \mu \mathrm{l}$ de sendas microdiluciones $\left(10^{-1}\right.$ a $\left.10^{-5}\right)$. Se incubó a $37^{\circ} \mathrm{C}$ durante 48 horas y se realizó el recuento; cuando éste fue superior a $10^{5} \mathrm{UFC} / \mathrm{ml}$ de aspirado duodenal, se consideró positivo para proliferación bacteriana (14).

Prueba de hidrógeno en el aliento. Al paciente se le tomó una muestra de aliento dos días después de realizada la endoscopia, utilizando los accesorios de Quintron e, inmediatamente, se le administraron $10 \mathrm{~g}$ de lactulosa (el contenido de un sobre de Lactulax ${ }^{\circledR}$, de Laboratorios Chalver) disueltos en $100 \mathrm{ml}$ de agua. Se determinó el hidrógeno en el aliento cada 15 minutos durante dos horas, utilizando el Microlyzer. Se consideraron positivos a los pacientes que presentaron un aumento de hidrógeno mayor de $20 \mathrm{ppm}$ con respecto al valor basal, en cualquiera de las mediciones realizadas durante la primera hora después del consumo de lactulosa (15).

Análisis estadístico. El procesamiento y el análisis de los datos se realizaron por medio del programa SPSS 11.5 para Windows en español. Con la ayuda del programa para análisis epidemiológico de datos tabulados, Epidat, versión 3.0 en español, se calculó la sensibilidad, la especificidad, los valores diagnósticos positivo y negativo, el índice kappa (16) y la prevalencia de proliferación bacteriana intestinal.

Consideraciones éticas. El consentimiento informado, que incluyó el nivel de riesgo, los beneficios, los procedimientos, el objetivo, la confidencialidad y la libertad de abandonar el estudio, fue aprobado por el Comité de Ética del Centro de Investigaciones de la Universidad Libre, Seccional Barranquilla.

\section{Resultados}

Hidrólisis de lactulosa comparada con la hidrólisis de lactosa por acción de la lactasa intestinal. La hidrólisis fue lineal a lo largo del tiempo; la de lactulosa alcanzó el 50\% cuando la incubación transcurrió durante 30 horas; en cambio, la hidrólisis de lactosa superó el $80 \%$ con sólo 4 horas de incubación y 12 veces menos de enzima. La acentuada diferencia entre la hidrólisis de lactosa y la de lactulosa indicó que esta última era mal sustrato para la enzima.

Cinética comparada de lactasa frente a lactosa y a lactulosa. En el cuadro 1 se presentan los resultados de la cinética. De la relación de los $\mathrm{K}_{\mathrm{m}}$ se infiere que la enzima fue 12 veces menos eficiente frente a lactulosa que frente a lactosa, $y$ de la relación de las $V_{\max }$ se deduce que la eficiencia de la enzima fue 20 veces menor con lactulosa. Por lo tanto, la enzima fue 240 veces menos eficiente frente a lactulosa, lo cual significa que este disacárido era un mal sustrato para la lactasa.

Recuento de bacterias a partir del cultivo de aspirado duodenal. De los 47 niños con patología gastrointestinal, $14(29,78 \%)$ presentaron recuentos positivos para proliferación bacteriana intestinal (media $=10^{\prime} 524.000 \mathrm{UFC} / \mathrm{ml}$ de aspirado duodenal) y los $33(70,21 \%)$ restantes presentaron recuentos negativos (media $=20.628 \mathrm{UFC} / \mathrm{ml}$ de aspirado duodenal). La prevalencia de proliferación bacteriana intestinal fue de $29,78 \%(28,6 \%$ a $30,9 \%)$. 
Cuadro 1. Comparación de la cinética de lactasa intestinal de mamífero frente a lactosa y frente a lactulosa.

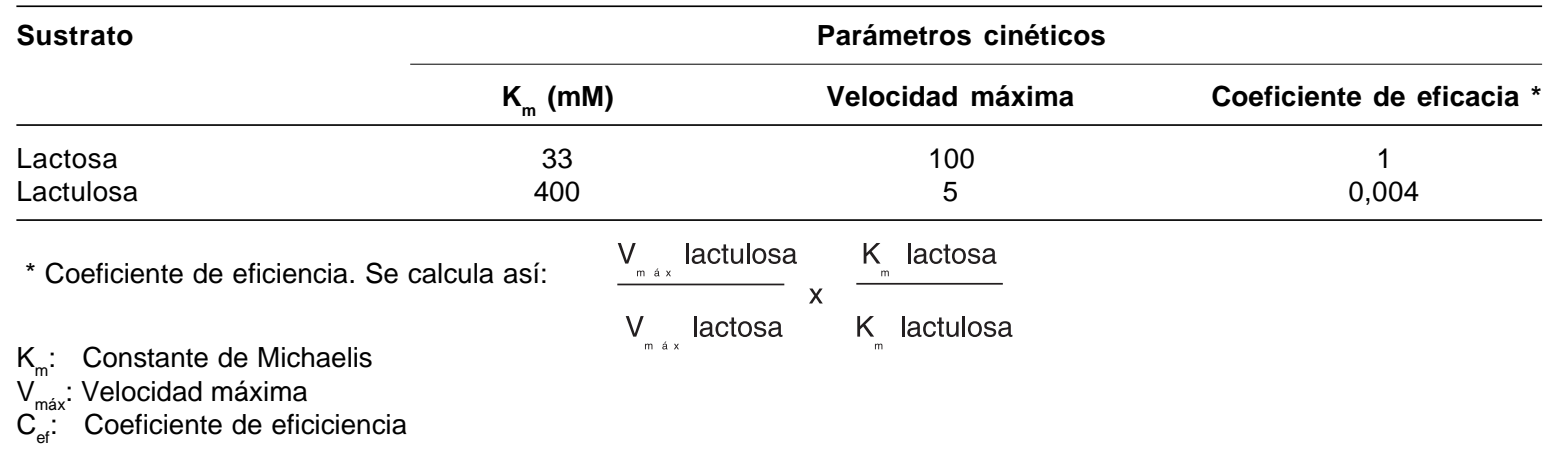

Prueba de hidrógeno en el aliento. La figura 1 muestra los promedios de hidrógeno en el aliento (ppm) a lo largo del tiempo, correspondientes a cada uno de los grupos en estudio. Se observa que los pacientes con proliferación bacteriana intestinal, diagnosticados mediante el patrón de referencia, presentaron un aumento de hidrógeno mayor de 20 ppm con respecto al valor basal en alguna de las mediciones realizadas durante la primera hora después del consumo de lactulosa, es decir, presentaron un aumento temprano; mientras que, en los pacientes sin proliferación bacteriana intestinal, el aumento de hidrógeno se presentó solo después de la primera hora, es decir, presentaron un aumento tardío.

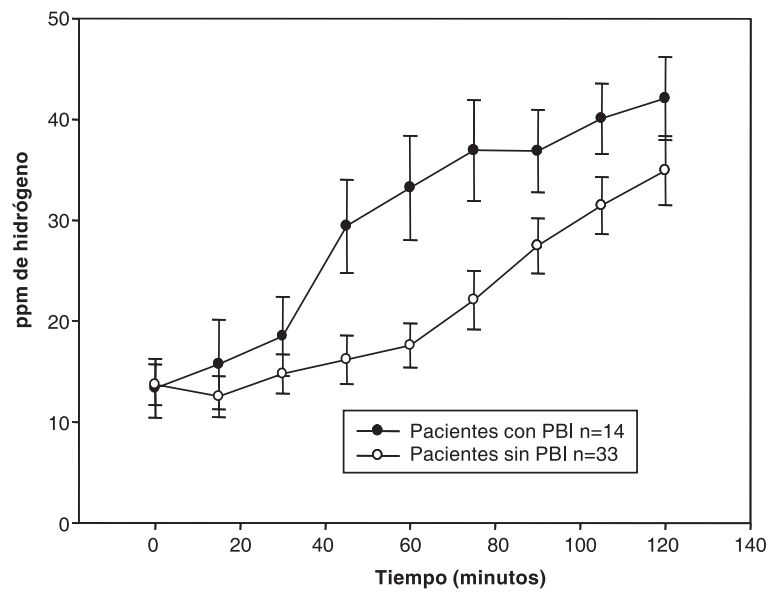

Figura 1. Comparación de la prueba de hidrógeno en el aliento entre pacientes con proliferación bacteriana intestinal (PBI) y pacientes sin ella. Se muestran los promedios y las desviaciones estándar de hidrógeno en el aliento correspondiente a las distintas mediciones a lo largo del tiempo.
Comparación entre la prueba del cultivo de aspirado duodenal y la prueba de hidrógeno en el aliento tras la administración oral de lactulosa. En el cuadro 2 se aprecian los datos que facilitaron la comparación entre las dos pruebas diagnósticas. De los 14 niños que resultaron positivos con el cultivo de aspirado duodenal, 12 también fueron positivos con la prueba de hidrógeno en el aliento; y de los 33 negativos frente al cultivo de aspirado duodenal, 30 fueron igualmente negativos con la prueba de hidrógeno en el aliento. La sensibilidad fue de $85,7 \%(82,0 \%$ a $89,4 \%)$ y la especificidad de $90,9 \%(89,3 \%$ a $92,5 \%)$. El valor diagnóstico positivo fue de $80 \%$ (76,5\% a $83,5 \%)$ y el valor diagnóstico negativo de $93,8 \%(92,1 \%$ a $95,3 \%)$. El índice kappa de 0,785 $(0,54-0,95)$ indicó importante concordancia entre las pruebas diagnósticas (16).

\section{Discusión}

En este estudio se reportan, por primera vez, los datos de la cinética comparada de lactasa intestinal en presencia de lactosa y de lactulosa; de ellos se colige que la lactasa intestinal es 240 veces menos eficiente frente a la lactulosa que frente a la lactosa. Los resultados de la cinética que aquí se reportan rectifican el dato publicado en 1965 por Dhalqvist y Gribosky (17); bajo condiciones experimentales precarias, puesto que estudiaron la hidrólisis con cromatografía en papel, encontraron que la lactasa intestinal no hidrolizaba la lactulosa.

En este hecho se apoyó Rodees, en 1979 (18), para plantear el empleo del disacárido en la prueba 
Cuadro 2. Relación entre los resultados obtenidos mediante la prueba de hidrógeno en el aliento y los resultados del cultivo de aspirado duodenal.

\begin{tabular}{|c|c|c|c|c|}
\hline & & \multicolumn{2}{|c|}{ Cultivo de aspirado duodenal } & \multirow[t]{2}{*}{ Total } \\
\hline & & Positivo & Negativo & \\
\hline \multirow{2}{*}{ Prueba de hidrógeno en el aliento } & Positivo & $12(85,7 \%)$ VP & $3(9,1 \%) \mathrm{FP}$ & 15 \\
\hline & Negativo & $2(14,3 \%) \mathrm{FN}$ & $30(90,9 \%) \mathrm{VN}$ & 32 \\
\hline Total & & 14 & 33 & 47 \\
\hline
\end{tabular}

VN: verdadero negativo, FN: falso negativo, VP: verdadero positivo, FP: falso positivo

de hidrógeno en el aliento. En este trabajo, midiendo enzimáticamente la galactosa liberada durante la hidrólisis, se demuestra que la lactulosa sí es hidrolizada, aunque pobremente; esto es, la afinidad de la lactasa de mamífero por la lactulosa es muy baja; la similitud entre la $\mathrm{K}_{\mathrm{m}}$ de la lactasa humana (30 mM) y la de lactasa de cordero (33 $\mathrm{mM}$ ) frente a la lactosa permite inferir que los resultados obtenidos con lactasa de cordero frente a la lactulosa se pueden extrapolar a la enzima humana; el pH óptimo similar $(\mathrm{pH}=5,8)$ de las dos enzimas frente a la lactosa así lo ratifica (datos sin publicar).

Pero lo esencial no cambia: la lactulosa es mal sustrato de la lactasa y, por ello, puede usarse como azúcar de carga en la prueba de hidrógeno en el aliento con alta probabilidad de que la aparición del pico precoz sea indicativa de resultado positivo para proliferación bacteriana intestinal $(1,19)$. Dicho de otra manera, es improbable que el pico precoz de hidrógeno, típico del individuo que tiene proliferación bacteriana intestinal, sea consecuencia de la acción de la lactasa sobre la lactulosa y no de la acción de la flora bacteriana irregularmente instalada en el intestino delgado, sobre dicho azúcar $(19,20)$; amén de la deficiencia de disacaridasas, entre ellas lactasa, que suele acompañar a la proliferación bacteriana intestinal $(1,7)$. Desde luego, la lactulosa no hidrolizada en el intestino delgado por la acción bacteriana, hará tránsito hasta el colon y allí será hidrolizada por la flora. Esta segunda hidrólisis es responsable del segundo pico de hidrógeno, pico tardío que suele aparecer en el registro gráfico de la prueba, pico que, inexorablemente, debe aparecer en las personas sanas (19).
La comparación de la prueba de hidrógeno en el aliento con la prueba bacteriológica valida el uso de la primera; su sensibilidad $(85,7 \%$ ) y su especificidad (90,9\%) así lo sustentan. La alta especificidad indica que la prueba tiene valor confirmatorio y el alto valor diagnóstico negativo indica que es alta la probabilidad de que, ante un resultado negativo, el paciente esté realmente sano. Por otro lado, el índice kappa $(0,785)$ demuestra que existe una importante concordancia entre las pruebas comparadas.

Los datos, en cuanto a especificidad, son semejantes a los reportados por Ghoshal et al. $(86 \%)$ (21) y Riordan et al. (70\%) (8), pero son discordantes en cuanto a la sensibilidad, a los datos presentados por ellos. Los aquí presentados son producto de un trabajo hecho con niños, mientras que sus trabajos eran con adultos.

La buena sensibilidad mostrada por la prueba de hidrógeno en el aliento, con lactulosa, indica que el azúcar, no digerido ni absorbido por los sujetos, era fermentado por la flora visitante y que el ambiente no era ácido para inhibir la producción de hidrógeno, al tiempo que la buena especificidad se puede deber a que la dosis de lactulosa suministrada es la adecuada para la acción de la flora bacteriana (19).

Por otro lado, la ausencia de estudios de proliferación bacteriana intestinal en las bases de datos exploradas e informaciones obtenidas a través de comunicaciones personales, inducen a pensar que esta condición clínica, en Colombia, podría estar subdiagnosticada. En cambio, sí se encuentran estudios con prueba de hidrógeno tras la administración de lactulosa, realizados en países vecinos: Arrieche et al. (22), estudiando 
niños caraqueños, reportaron una prevalencia de $82,9 \%$ en un grupo cuyas formas clínicas de presentación más frecuentes fueron dispepsia y dolor abdominal; Dos Reis et al. (23) informaron una prevalencia de proliferación de $6 \%$ en niños brasileños, sanos y asintomáticos; $y$, Torres et al. (24), en el Perú, dieron cuenta de una prevalencia de $24,1 \%$ en pacientes adultos con diarrea crónica y de $12,5 \%$ en sujetos controles normales.

La prevalencia hallada en el presente estudio realizado con niños que habían consultado por trastornos gastrointestinales, como dolor abdominal, flatulencia, sensación de plenitud, vómitos y diarrea, fue de $29,78 \%(28,6 \%$ a $30,9 \%$ ). Esto concuerda con los datos antes reseñados y plantea la inquietud de estudiar, más adelante, si la aclorhidria asociada con la infección por Helicobacter pylori o si la alta contaminación bacteriana ambiental, propia de países subdesarrollados, podrían explicar la prevalencia hallada. De cualquier manera, la prevalencia no sólo depende de la población (etnia, cultura, edad) sino del tipo de prueba diagnóstica aplicada; aun usando pruebas de aliento, dependerá del tipo y la dosis de sustrato usado.

En el futuro, sería interesante, por un lado, experimentar con dosis más bajas de lactulosa, de tal manera que sólo se visualice un pico: el precoz, en casos positivos, y el tardío, en casos negativos; y por el otro, explorar la bondad del uso de otros análogos de lactosa como lactitol, 3-metil-lactosa y galactosil-xilosa. En lo atinente a la prevalencia de proliferación bacteriana intestinal y su correspondencia con la clínica, el asunto será objeto de una publicación diferente.

En conclusión, este estudio aporta evidencia experimental que le da sustento al empleo de lactulosa en la prueba de hidrógeno en el aliento para diagnosticar proliferación bacteriana intestinal y, además, permite validar la prueba de hidrógeno en el aliento posterior a la ingestión de lactulosa, como prueba tamiz. Entonces, por sus fundamentos, por su simplicidad, por su carácter no invasivo y por su alta especificidad, la prueba de hidrógeno en el aliento es una buena alternativa como prueba en el diagnóstico de proliferación bacteriana intestinal.

\section{Conflicto de intereses}

Laboratorios Chalver de Colombia fue ajeno al diseño y a la financiación del estudio, su participación se limitó a la donación de su producto Lactulax® (lactulosa).

\section{Financiación}

Esta investigación fue financiada por el rubro de investigaciones del presupuesto del 2005 de la Universidad Libre, Seccional Barranquilla.

\section{Agradecimientos}

A la Universidad Libre por la financiación del proyecto, a Laboratorios Chalver de Colombia por proveer gratuitamente la lactulosa que se usó para la prueba de hidrógeno en el aliento y a Jorge Luis Bilbao por las atinadas observaciones formuladas después de la lectura crítica del manuscrito.

\section{Referencias}

1. Casellas F. Manejo del sobrecrecimiento bacteriano. Estrategias clínicas en digestivo. Prous Science S.A. Web médica acreditada. Barcelona. 2002. [Consultado: 6 de julio de 2006]. Disponible en: http://www.prous.com/ digest/protocolos/view_protocolo.asp?id_protocolo=2.

2. Casellas F. First principles of gastroenterology: the basis of disease and approach to management. En: Bacterial overgrowth syndrome. First edition. Canadá: AztraZeneca; 1999. p.251-3.

3. Omoike IU, Abiodun PO. Upper small intestinal microflora in diarrhea and malnutrition in Nigerian children. J Pediatr Gastroenterol Nutr. 1989;9:314-21.

4. Quera R, Quigley E, Madrid A. Small intestinal bacterial overgrowth. An update. Rev Med Chil. 2005;133: 1361-70.

5. Gregg CR. Enteric bacterial flora and bacterial overgrowth syndrome. Semin Gastrointest Dis. 2002;13:200-9.

6. Guarner F, Malagelada JR. Gut flora in health and disease. Lancet. 2003;361:512-9.

7. Husebye E. The pathogenesis of gastrointestinal bacterial overgrowth. Chemotherapy. 2005;51 (Suppl.1):1-22.

8. Riordan SM, McCalver CJ, Walker BM, Ducombe VM, Bolin TD, Thomas MC. The lactulose breath hydrogen test and small intestinal bacterial overgrowth. Am J Gastroentol. 1996;91:1795-803.

9. Alves GM, de Moraes BM, Fagundes-Neto $U$. Nutritional status and breath hydrogen test with lactose 
and lactulose in Terena Indian Children. J Pediatr (Rio J). 2002;78:113-9.

10. Gisbert JP, González-Lama Y. Breath tests in the diagnosis of gastrointestinal diseases. Gastroenterol Hepatol. 2005;28:407-16.

11. Skovbjerg $\mathbf{H}$, Noren $\boldsymbol{H}$. Purification and characterization of amphiphilic lactase fhlorizin hydrolase. Eur J Biochem. 1981;114:653-61.

12. Duodoroff M. D-Galactose dehydrogenase of Pseudomonas saccaharophila. En: Methods in enzymology. Volumen V. New York: Academy Press; 1962. p.339.

13. Lowry OH, Rosebroug NJ, Farr AL, Randall RJ. Protein measurement with the Folin phenol reagent. J Biol Chem. 1951;193:265-72.

14. Ghoshal U, Ghoshal UC, Ranjan P, Naik SR, Ayyagari A. Spectrum and antibiotic sensitivity of bacteria contaminating the upper gut in patients with malabsorption syndrome from the tropics. BMC Gastroenterol. 2003;3:9.

15. Hamilton LH. Breath tests and gastroenterology. Second edition. Milwaukee: QuinTron Instruments Company; 1998. p.39-49.

16. Rey Calero J, Herruzo R, Rodríguez F. Fundamentos de epidemiología clínica. Madrid: Síntesis; 1996. p.2535.

17. Dahlqvist A, Gribosky J. Inability of the human small intestinal lactase to hydrolyse lactulose. Biochim Biophys Acta. 1965;110:635-6.
18. Rhodes JM, Middleton P, Jewell DP. The lactulose hydrogen breath test as a diagnostic test for smallbowel bacterial overgrowth. Scand J Gastroenterol. 1979;14:333-6.

19. Bishop WP. Breath hydrogen testing for small bowel bacterial overgrowth - A lot of hot air? J Pediatr Gastroenterol Nutr. 1997;25:245-6.

20. Pimentel M, Chow EJ, Lin HC. Eradication of small intestinal bacterial overgrowth reduces symptoms of irritable bowel syndrome. Am J Gastroenterol. 2000;95:3503-6.

21. Ghoshal UC, Ghoshal U, Das K, Misra A. Utilility of hydrogen test in diagnosis of small intestinal bacterial overgrowth in malabsorption syndrome and its relationship with oro-cecal transit time. Indian $\mathrm{J}$ Gastroenterol. 2006;25:6-10.

22. Arrieche M, Hernández D, Olza M, Pestana E, Morera C, Jaen D. Sobrecrecimiento bacteriano en el intestino delgado: presentación clínica en la edad pediátrica. Colombia Médica. 2005;36(Suppl.1):58-87.

23. Reis JC, Morais MB, Fagundes-Neto U. Teste do H2 no ar expirado na avaliaçäo de absorçäo de lactose e sobrecrescimento bacteriano no intestino delgado de escolares/Breath hydrogen test to evaluate lactose absorption and small bowel bacterial overgrowth in children. Arq Gastroenterol. 1999;36:169-76.

24. Torres R, León R, Berendson R, Curioso W, Roe E, Biber M. Sobrecrecimiento bacteriano en el intestino delgado en pacientes con diarrea crónica y sujetos controles normales. Rev Gastroenterol Perú. 2003;23:111-4. 